\title{
Berger, Pascal: Religion und Wirtschaft bei Georg Simmel. Über die Chancen und Grenzen ganzheitlicher Lebensführung, 606 S., transcript, Bielefeld 2019.
}

\author{
Oliver Hidalgo \\ Online publiziert: 29. September 2020 \\ (C) Der/die Autor(en) 2020
}

Hinter dem eher harmlos anmutenden Titel der zu besprechenden Monografie steckt - entgegen des ersten Eindrucks des Rezensenten - keine Randnotiz zu einer Themenverknüpfung, die der Soziologenpapst Georg Simmel im Rahmen seines vielschichtigen Werks eher nebenbei abgehandelt hätte, sondern nicht weniger als der Versuch, eine neue und frische Lesart von Simmels Gesamtwerk zu wagen. In dieser Hinsicht stellt die Dissertation von Pascal Berger die reizvolle These in den Raum, dass es nach Simmel eigentlich erst das Geld ist, das als Realisierung und Materialisierung des „Vitaldualismus aus individuellem Leben und überindividueller Form“ (S. 11) unter den säkularen Bedingungen der Moderne ein religiöses Leben gestattet. Über die Nachzeichnung einer signifikanten Wechselwirkung zwischen Religion und Wirtschaft hinausgehend, avanciert das Geld dabei zum Symbol dafür, dass Gott nunmehr im Individuum ,wohnt“ (S. 12), und trägt somit dem Umstand Rechnung, dass eine jenseits des Lebens, das heißt in der Transzendenz verortete Gottheit den Konditionen der Moderne unweigerlich zuwiderläuft.

Mit jener komplexen Interpretation, die er bis in die Kapillaren von Simmels Kultur- und Lebensphilosophie beziehungsweise -soziologie verfolgt, spricht sich Berger gegen vordergründige Analogien aus, die im Anschluss an Walter Benjamin das Geld oder das ökonomische Kapital zum, Gott der Moderne ‘ küren oder wie Max Webers berühmte Protestantismusthese umgekehrt die Religion als kausalgenetische Grundlage einer umfassend rationalisierten Geld- und Kapitalwirtschaft identifizieren. Stattdessen wird die Option religiöser Lebensführung als solche ins Zentrum von Simmels Theorien und Überlegungen gerückt und eng an die Herausdifferenzierung der Geldwirtschaft gekoppelt, die das Individuum erst von seiner ,urwüchsigen Verflechtung mit der Ökonomie“ (ebd.) emanzipiert. Dies versetze den Einzelnen im Gegenzug in die Lage, sich ,frei', das heißt in bewusster Eigenselektion in die Formen der Gesellschaft und Kultur zu integrieren. Die Überindividualität des Geldes ,erlöst' das Individuum hier förmlich von seinen traditionellen Bindungen und substituiert in dieser Weise das Gottessymbol. Das einstige religiöse Sinnbedürfnis mutiert zugleich zu einer Sehnsucht nach Einheit, die das Individuum in der sä-

O. Hidalgo $(\square)$

Westfälische Wilhelms-Universität Münster, Münster, Deutschland

E-Mail: hidalgoo@uni-muenster.de 
kularen Moderne nicht mehr außerhalb seiner selbst finden kann, weshalb es sich überindividuell festgelegten, dem individuellen Leben äußerlich bleibenden Inhalten entzieht.

Bergers lebensphilosophischer Argumentation gelingt es, eine bislang eher verborgene, positive Kehrseite der in Simmels „Philosophie des Geldes“ eruierten, alle alternativen normativen Werte nivellierenden Kraft (die am Ende nichts als die Totalität des Geldes gelten lässt) zu rekonstruieren. Die Inhaltslosigkeit, ja reine Form des Geldes vermag unter diesen Vorzeichen den Wunsch nach religiöser Vollendung und Geschlossenheit zu erfüllen, ohne selbst notgedrungen zum Fetisch zu werden. Bisweilen ist dabei zwar eine gewisse Überinterpretation der eigenen These festzustellen, etwa wenn Berger das apriorische Formungsprinzip der Vergesellschaftung, welches Simmel im Anschluss an Immanuel Kants Transzendentalphilosophie entfaltet, dafür verantwortlich macht, dass sich in der Soziologie keine ,SimmelSchule“ etablieren konnte (S. 577) - und das, obwohl dafür bekanntermaßen weit ,profanere ' Gründe ursächlich sind, wie der im Kaiserreich verbreitete Antisemitismus, der Simmels akademische Karriere entscheidend behinderte. Dennoch zeichnet die Studie eine insgesamt sehr überzeugende Gedankenführung aus, die dem Leser manches Rätsel seiner bisherigen Simmel-Lektüre zu lösen vermag.

Für Sozialwissenschaftler von nachhaltigem Erkenntniswert dürften zudem vor allem die en passant (zum Beispiel S. $233 \mathrm{ff} ., 413$ ) sowie am Ende des Buchs lancierten Ausblicke auf die Beziehungs- und Trennlinien sein, die sich zwischen den soziologischen Klassikern Émile Durkheim, Max Weber und Georg Simmel im Hinblick auf die aufwendig verhandelte Fragestellung ausfindig machen lassen. Mehr hätte man hingegen gern über den Begriff „säkular-religiöses Leben“ erfahren, den Berger zu Beginn seiner Studie einführt, ohne genauer zu erläutern, wie dieses offenkundige Oxymoron letztlich zu verstehen sei: Als (bloße) Verweltlichung und/oder Immanentisierung des Göttlichen? Als semantische und/oder substanzielle Überlappung zwischen dem Säkularen und Religiösen? Als traditionelle Opposition, die in der Moderne nach Simmel zum Erliegen kommt? Vielleicht gar als Einheit oder Zusammenfall der Gegensätze auf höherer Ebene, gleich einer coincidentia oppositorum im Anschluss an Cusanus mitsamt ihren evidenten Ausgreifungen in Richtung des Pantheismus?

Die Aussparung solcher und anderer über das Werk Simmels hinausweisenden Klärungen suggeriert gleichermaßen die Stärke und Schwäche von Bergers Buch: Für Kenner der Materie ist die Studie mit Sicherheit ein Gewinn, weil sie tiefe Einblicke in den werkimmanenten Zusammenhang der oftmals recht heterogen anmutenden, eher unsystematisch angelegten Schriften Simmels gewährt und diesbezüglich auch mit manchem Fundstück aufwarten kann (etwa was den Zeitpunkt seiner Wende von der Lebenspsychologie zur Lebensphilosophie betrifft). Für Leser, die sich indes weniger für Simmels Stellung im Kanon der Soziologie als für die gesellschaftspolitischen Implikationen seiner religions- und wirtschaftstheoretischen Überlegungen und nicht zuletzt seine Relevanz für aktuelle Debatten über die religiöse Bedeutung des Geldes interessieren, bietet das Buch jedoch nicht nur ziemlich schwere Kost, sondern auch verhältnismäßig wenig Aufschlussreiches. Ein Scharnier, wie jene beiden gegenläufigen Belange eventuell miteinander in Einklang gebracht werden könnten, liefert Berger immerhin mit, wenn er die „funktionale Äquivalenz“ (S. 15) 
zwischen Religion und Wirtschaft im Gefolge der Luhmannschen Systemtheorie als Gedanken erwähnt, der sich in dieser Hinsicht weiter ausführen ließe (vgl. S. 380 ff.). Die monierte Trennschärfe zur abgelehnten These „Geld als Gott“ müsste hierfür allerdings entweder spezifiziert oder aufgegeben werden.

Funding Open Access funding enabled and organized by Projekt DEAL.

Open Access Dieser Artikel wird unter der Creative Commons Namensnennung 4.0 International Lizenz veröffentlicht, welche die Nutzung, Vervielfältigung, Bearbeitung, Verbreitung und Wiedergabe in jeglichem Medium und Format erlaubt, sofern Sie den/die ursprünglichen Autor(en) und die Quelle ordnungsgemäß nennen, einen Link zur Creative Commons Lizenz beifügen und angeben, ob Änderungen vorgenommen wurden.

Die in diesem Artikel enthaltenen Bilder und sonstiges Drittmaterial unterliegen ebenfalls der genannten Creative Commons Lizenz, sofern sich aus der Abbildungslegende nichts anderes ergibt. Sofern das betreffende Material nicht unter der genannten Creative Commons Lizenz steht und die betreffende Handlung nicht nach gesetzlichen Vorschriften erlaubt ist, ist für die oben aufgeführten Weiterverwendungen des Materials die Einwilligung des jeweiligen Rechteinhabers einzuholen.

Weitere Details zur Lizenz entnehmen Sie bitte der Lizenzinformation auf http://creativecommons.org/ licenses/by/4.0/deed.de.

\section{Willke, Helmut: Komplexe Freiheit. Konfigurationsprobleme eines Menschenrechts in der globalisierten Moderne, 308 S., transcript, Bielefeld 2019.}

\section{Florian Eyert · Florian Irgmaier}

Online publiziert: 28. Juli 2020

(C) Der/die Autor(en) 2020

Freiheit und ihre politischen Bedingungen sind in den letzten Jahren erneut zu einem zentralen Gegenstand sozialwissenschaftlicher Auseinandersetzung geworden. Allerorts lassen sich Gefährdungen des Liberalismus ausmachen, vom Populismus über

F. Eyert $(\bowtie) \cdot$ F. Irgmaier

Weizenbaum-Institut für die vernetzte Gesellschaft/Wissenschaftszentrum Berlin für Sozialforschung, Berlin, Deutschland

E-Mail: florian.eyert@wzb.eu

F. Irgmaier

E-Mail: florian.irgmaier@wzb.eu 